\title{
ENFERMERÍA EN EL ROL DE GESTORA DE LOS CUIDADOS
}

\author{
NURSING IN THE ROLE OF HEALTH CARE MANAGER
}

\author{
Silvana Estefo AgÜero* \\ Tatiana Paravic Klijn ${ }^{* *}$
}

\begin{abstract}
RESUMEN
En Chile, según lo establecido en el Código Sanitario en 1997 y en la Norma General Administrativa N$^{\circ} 19$ del año 2007, la enfermera es la responsable de la gestión del cuidado, es decir, debe asumir el rol de gestora de los cuidados y como tal se le atribuye una serie de funciones y atribuciones. El objetivo de este artículo es destacar la importancia de algunos aspectos necesarios para ejercer el rol de gestora de los cuidados de enfermería, en especial la importancia del liderazgo, y en este punto la habilidad de comunicación y la capacidad de toma de decisiones. Se plantea cómo cada uno de estos aspectos influye en el aseguramiento de la calidad de los cuidados otorgados, al igual que la continuidad de los mismos.
\end{abstract}

Palabras clave: Enfermería, gestión del cuidado, líder.

\begin{abstract}
In Chile, as established by the 1997 Sanitary code and the 2007 General Administrative Law $\mathrm{N}^{\circ} 19$, the nurse is responsible for the management of care. That is to say, nursing must assume the role of patient care manager and as such, many duties and responsibilities are assigned to the nurse. The purpose of this article is to bring attention to the importance of demonstrating the necessary aspects of the role of nursing care manager, especially the importance of leadership and the ability to communicate and make decisions. It is shown that many of these dimensions influence the quality of care provided as well as the continuity of care.
\end{abstract}

Key words: Nursing, care management, leader.

Fecha recepción: 02/11/2009 Fecha aceptación: 10/08/2010

\section{INTRODUCCIÓN}

En Chile se asigna la responsabilidad legal, ética y social al profesional de enfermería en relación a la gestión del cuidado, esto nos obliga a asumir un compromiso y una responsabilidad: asegurar la continuidad y calidad de los cuidados.

Si bien a través de la historia en el profesional de enfermería siempre ha estado pre- sente el otorgar cuidados de calidad, ahora existe un reconocimiento legal a nuestra profesión y como tal debemos evidenciar nuestro rol de gestora de los cuidados. El presente artículo tiene como objetivo abordar aspectos importantes que debe desarrollar y poseer el profesional de enfermería: liderazgo, habilidad de comunicación y toma de decisiones, y cómo éstos se relacionan con la calidad de los cuidados otorgados.

En primer lugar, se abordará lo que diver-

\footnotetext{
*Enfermera. Docente Depto. de Enfermería, Universidad de Magallanes. E-mail: silestefo@udec.cl

${ }^{* *}$ Enfermera. Docente Depto. de Enfermería, Universidad de Concepción, Chile. E-mail: tparavic@udec.cl
} 
sos autores entienden por el término "cuidado", y la "gestión del cuidado" según las normativas vigentes en Chile, con algunas de las funciones y atribuciones que se desprenden de ello. Por último, se abordará el liderazgo como componente esencial del rol de gestora de los cuidados y los elementos fundamentales para el ejercicio de éste.

\section{CUIDADO}

El cuidado es inherente al ser humano, es la acción que preserva nuestra especie. El ser humano desde antes del nacimiento cuida a sus semejantes y también durante todo su crecimiento y desarrollo (1), es así como desde la década de los años sesenta el término cuidado se ha venido usando en la literatura. Al revisar ésta, se encuentra una distinción entre el término care y caring, distinción que se hace necesaria en enfermería. El término "care" se refiere a la acción de cuidar, según esta definición todo ser humano puede cuidar, y "caring" incorpora además un sentido y una intencionalidad humanitaria en esta acción. En el contexto de enfermería es usado para designar "los cuidados profesionales que la enfermera lleva a cabo y que suponen un compromiso humanista y un interés por sus semejantes" (2).

Diversas teóricas de enfermería han definido el término cuidado. Es así como Leinninger (citado por Kerouac) plantea que "el cuidado es la esencia y el fenómeno central de la enfermería y que debe ser valorizado en todas las áreas de su ámbito profesional" (3).

Watson enfatiza que "el cuidado percibe los sentimientos del otro y reconoce su condición de persona única" (4). El término cuidado para la enfermería debe verse desde varias dimensiones y perspectivas. Medina plantea que "el concepto de cuidado se puede ver desde tres percepciones: emocional, transaccional y de desarrollo y existencial"
(2). Desde el contenido emocional de las interrelaciones enfermera-paciente, Benner y Wrubel plantean que el cuidado es "cuidar o tomar contacto, es el elemento esencial en la relación enfermera-paciente y la enfermera debe tener la capacidad para empatizar o sentir desde el paciente; representa la principal característica de la relaciones profesionales"(5), es establecer una relación de ayuda, comprometerse emocionalmente con el otro, ya que es la única manera de que la relación sea significativa, de que vea a ese ser humano que necesita de la ayuda profesional en su totalidad y que él vea a una enfermera cálida, empática, que no lo juzga sino que le brinda la ayuda profesional que necesita y que, al término de esta relación, ambos participantes, enfermera-paciente, saldrán fortalecidos y capaces de seguir estableciendo relaciones significativas (6).

Desde una segunda perspectiva, el sentido transaccional y de desarrollo, es donde la enfermera obtiene crecimiento personal a partir de su relación con el enfermo. Planner (citado por Medina) afirma que cuidado "es un proceso interactivo por el que la enfermera y el cliente se ayudan mutuamente para desarrollarse, actualizarse y transformarse hacia mayores niveles de bienestar. El cuidado alcanza una apertura del yo al otro, una determinación sincera de compartir emociones, ideas, técnicas y conocimientos" (2); aquí la enfermera debe demostrarse tal cual es, auténtica, no temer a demostrar sus sentimientos ni a mostrarse como una persona capaz de expresar emociones y compartir su vida con otra persona que necesita de su ayuda profesional.

Desde la tercera perspectiva, la existencial, Mayeroff (citado por Medina) determina los atributos generales del cuidado: "Cuidar es la antítesis del uso simplista de otra persona para satisfacer nuestras necesidades, el cuidado debe ser entendido como ayuda a otra persona que permite el desarrollo, de la misma manera que la amistad aparece a través de la confianza mutua que produce 
una transformación profunda y cualitativa de las relaciones"(2). En esta perspectiva lo esencial es trascender al otro a través del establecimiento de relaciones significativas en la entrega del cuidado.

Siguiendo con el proceso de conceptualización del cuidado también se han identificado "dimensiones esenciales en el acto de cuidar como es la interacción, la participación y el afecto" (7).

Hay autores (7-9) que enfatizan que el concepto de interacción es el elemento más esencial del cuidado. En relación a la dimensión participación del paciente en su cuidado, Orem (10) y Pender (11) principalmente plantean que las enfermeras deben promover activamente la participación del individuo tanto en su autocuidado como en la toma de decisiones. En cuanto al afecto, ésta es una de las dimensiones del cuidado que contribuyen a la satisfacción tanto del paciente como de la enfermera (7). García y Romero, plantean que "el afecto se identifica como una vivencia humana referida al tono emocional y al estado de ánimo, evidenciándose en los sentimientos y emociones de cada persona, imprimiéndole un carácter humano y terapéutico a la vez" (12).

Si bien es importante los esfuerzos que se han realizado para llegar a conceptualizar el término cuidado, Daza de Caballero, Torres y Prieto de Romano plantean que "por ser el cuidado de enfermería un acto que implica el abordaje integral de la persona, el tratar de establecer límites precisos entre sus dimensiones es una tarea inútil que impide apreciar la riqueza del acto mismo del cuidar" (7).

\section{GESTIÓN DEL CUIDADO}

En Chile el 16 de diciembre de 1997 se incorporó en el Código Sanitario, Libro V “del ejercicio de la medicina y profesiones afines", el artículo 113, donde se reconoce a la Enfermería como profesión. Éste declara que "los servicios profesionales de la enfermera comprenden la gestión del cuidado en lo relativo a promoción, mantención y restauración de la salud, la prevención de enfermedades o lesiones, y la ejecución de acciones derivadas del diagnóstico y tratamiento médico y el deber de velar por la mejor administración de los recursos de asistencia para el paciente" (13). La Gestión del Cuidado de Enfermería en Chile es "entendida como el ejercicio profesional de la enfermera sustentada en su disciplina, la ciencia de cuidar, se define como la aplicación de un juicio profesional en la planificación, organización, motivación y control de la provisión de los cuidados, oportunos, seguros e integrales, que aseguren la continuidad de la atención y se sustenten en las políticas y lineamientos estratégicos de la institución" (14). En este sentido, la gestión del cuidado debe "permitir brindar un cuidado planificado, utilizando los recursos disponibles, fijando objetivos, metas y estrategias" (15); además implica que sea un proceso creativo, movilizando los recursos humanos y los del entorno con la intención de mantener y favorecer el cuidado de la persona (3), debe estimular la creación de modelos de cuidado basados en la concepción de la disciplina de enfermería, el uso de un lenguaje común, de símbolos, de conceptos comunes en la práctica clínica que refuercen el potencial, el compromiso y la identidad profesional de la enfermería, pero, por sobre todo, debe asegurar la calidad del cuidado que se otorga a los usuarios y a su familia" (16).

Según el artículo 113 del Código Sanitario (13) y la Norma General Administrativa $N^{\circ} 19$, "la gestión del cuidado debe estar a cargo de un profesional enfermera/o, con formación y competencias técnicas y de gestión en el área de la gestión de los cuidados, además se le dan atribuciones para organizar, supervisar, evaluar y promover el mejoramiento de la calidad de los cuidados de enfermería, a fin de otorgar una atención segura, oportuna, continua, con pertinencia 
cultural y en concordancia con las políticas y normas establecidas por el Ministerio de Salud" (14); desde este punto de vista, "la gestión del cuidado es intransferible ya que requiere de un criterio profesional que, desde su planificación hasta su ejecución, responda a estándares éticos, jurídicos y científico-técnicos, que sólo se alcanzan con una formación superior impregnada de la tradición y evolución histórica de la enfermería, la gestión sólo será delegable a otra enfermera/o" (17). La enfermera es la responsable de la gestión del cuidado y en la literatura a esta responsabilidad se le define como el rol de gestora de los cuidados. Diversos autores han definido lo que implica este rol de gestora, es así como Meleis plantea que la enfermera gestora de los cuidados tiene "la responsabilidad de crear una cultura de organización que favorezca la práctica de los cuidados, seleccionar el personal con formación, desarrollar la capacitación y la implementación de un modelo para guiar la práctica de enfermería"(16), debe "apoyar al personal cuidador en su labor de cuidar en forma individualizada al paciente y su familia" (18), considerando siempre los "valores, actitudes y conocimientos de la disciplina que le brindan una visión distinta y específica de la gestión de los cuidados" (16); debe ejercer un liderazgo, el que "debe ser comprensivo, generando un clima de trabajo favorable, participativo, fomentando el trabajo en equipo, haciendo partícipe a su personal en las decisiones" (3).

Es así como en este rol de gestora surge inevitablemente el término líder. El liderazgo es un componente esencial de la gestión, para lo cual las enfermeras deben prepararse. Una de las habilidades que las autoras consideran primordial para lograr el éxito en el rol de líder como gestora del cuidado en enfermería es la habilidad de comunicación, un término muy desarrollado en lo teórico pero muy poco utilizado en la práctica. La habilidad para comunicarse es el núcleo del liderazgo (19), por lo tanto "la comunicación constituye un elemento fundamental en el proceso de ejercer el liderazgo en enfermería, ya que la forma en que se transmite el mensaje interferirá con el resultado deseado" (20). En este sentido, "la comunicación es la base de liderazgo, porque el liderazgo es una relación interpersonal en la que los líderes influyen en las personas a cambiar a través del proceso de comunicación. El éxito del líder está relacionado con su capacidad para comunicarse con los demás, este proceso implica el uso sistemático de símbolos para transmitir información y llegar a un entendimiento acerca de una situación. El objetivo del comunicador es transmitir un mensaje a otra persona, de manera que el mensaje se reciba como está previsto, sin distorsión" (19). La comunicación es uno de los más influyentes, importantes y complejos aspectos de las conductas de las personas. La actividad cotidiana de un profesional como líder en la gestión del cuidado de enfermería está llena de actividades en las que la comunicación es imprescindible, tanto en los aspectos verbales y no verbales de la comunicación. Más que explicar el proceso de comunicación, creemos importante abordar algunos elementos necesarios que deben estar presentes en la comunicación. Uno de ellos es la escucha activa, "escuchar activamente es poner todos los medios a nuestro alcance para lograr entender lo que nos quieren decir, mostrando además un afán de interés ante la presencia del mensaje y una conducta de colaboración con el emisor en su tarea de comunicar" (21); escuchar es una de las mejores formas de obtener la mayor cantidad de información, además de demostrar el interés por el otro, es también interpretar lo escuchado, evaluarlo y responder a la información transmitida. No basta escuchar a otra persona para que ella sea consciente de que la escuchan, es necesario transmitirlo, comunicar con actos conscientes al otro que 
hemos prestado atención a sus palabras, es la habilidad de captar y reflejar el mensaje del otro. El mensaje se debe reformular con las propias palabras del receptor, pero no sólo su contenido (percepción denotativa), sino también los sentimientos y actitudes que lo acompañan (percepción connotativa). No es una habilidad muy frecuente puesto que no se suele enseñar y se practica poco, y sin embargo es la más valorada por las personas con las que uno interactúa, la escucha activa se relaciona con la empatía, vista como la aptitud de ponerse en el lugar del otro, siempre considerando su cultura, sus valores, su individualidad, de esta manera no sólo percibimos las palabras sino también a la persona en su esencia. Teniendo presente estos elementos en la comunicación, nos permitirá "garantizar que cada profesional reciba la información que necesita para realizar su trabajo, se pueda comunicar con facilidad con otros profesionales con los que interacciona y con los que participa en el cuidado del paciente y pueda aportar sus opiniones y su conocimiento cuando se detecten problemas que se quieran solucionar" (22). "La comunicación eficaz facilitará a la enfermera gestora de los cuidados el trabajo entre profesionales de distintas disciplinas, ya que la calidad de la atención que recibe el paciente es el resultado de un proceso en el que tienen que intervenir muchas personas y debe hacerlo en forma coordinada. En una organización la comunicación es esencial para el ejercicio de influencia sobre la coordinación de las actividades de grupo y para garantizar el proceso de liderazgo" (23).

Otra de las capacidades que consideramos primordial al ejercer el rol de líder en la gestión de los cuidados en enfermería es la capacidad de tomar decisiones. Una de las principales funciones de los profesionales que ejercen una responsabilidad directiva, o más propiamente gestora, se centra en la toma de decisiones. Éste es un proceso nada fácil, que compromete el quehacer directivo de forma continúa (18), debiendo seleccionar entre las alternativas precisas en la toma de decisión (24). Es así que para tomar una decisión se requiere percibir diversas opciones, significa decidir o elegir entre varias alternativas, aunque éstas sean las más elementales o primarias; sí o no, actúo o no actúo, dejo las cosas como están o hago un cambio (25). "La toma de decisiones es un proceso reflexivo e intelectual conformado por unas secuencias que pueden ser analizadas, perfeccionadas e integradas, con objeto de conseguir una mayor precisión y exactitud en la solución de los problemas y en el inicio de acciones" (26), incluye identificar un problema, buscar soluciones y seleccionar la alternativa que mejor permita alcanzar el objetivo de quien toma la decisión (27). El líder, en este caso la enfermera gestora de los cuidados cuando toma una decisión correcta, consigue algo más que resolver los problemas inmediatos, consigue a largo plazo fomentar la toma de decisiones acertada por parte de las otras enfermeras, a través de la observación de su rol de líder. (24). La solución de problemas es una capacidad que puede perfeccionarse, es decir la habilidad de decidir se puede aprender (25), por lo que consideramos primordial considerar desarrollar esta capacidad en la formación del recurso humano del profesional de enfermería. Si revisamos la literatura, existen diversos modelos organizativos para tomar decisiones: el modelo racional, modelo político, modelo colegial y modelo burocrático, modelo del cubo de basura, por mencionar algunos, pero, independiente del modelo que se adopte, lo importante es desarrollar el pensamiento crítico, ya que éste contribuye a la calidad de la toma de decisiones y a la solución de problemas (24). El profesional de enfermería debe estar preparado en los diversos aspectos que involucra la gestión de los cuidados, para responder con una atención de calidad ante los usuarios que demandan salud, y ante la sociedad que nos ha otorgado tal responsabilidad. 


\section{CONCLUSIÓN}

En Chile, a través del artículo 113 y la normativa administrativa $\mathrm{N}^{\circ} 19$, se le confiere a la enfermera la responsabilidad de "la gestión del cuidado", esto implica una serie de funciones, atribuciones y responsabilidades, donde el rol de la enfermera como gestora del cuidado cobra importancia y a la vez desafíos. Uno de estos desafíos es que debe asumir el liderazgo en esta gestión y como líder debe tener habilidades de comunicación, y tener información y conocimiento para la correcta toma de decisiones. Esto permitirá a la enfermera gestora del cuidado asegurar que todas las personas involucradas en el cuidado del paciente obtengan la información necesaria para la continuidad y calidad de los cuidados que necesiten. Para ello el profesional de enfermería debe estar muy preparado en estos aspectos y las escuelas de Enfermería tienen una responsabilidad en la formación del recurso humano de enfermería, el cual debe ser capaz de responder a los desafíos de nuestro rol de gestora de los cuidados de salud de la población.

\section{REFERENCIAS}

1. Landeros-Olvera E, Salazar T, Reflexiones sobre el cuidado. Rev. Enferm. Inst Mex Seguro. 2006; 14 (3): 121-122.

2. Medina J. Pedagogía del cuidado: saberes y prácticas en la formación universitaria. Barcelona. Editorial Laertes; 1999.

3. Kerouac S, Pepin J, Ducharme F, Duquette A, Major F. El pensamiento enfermero. Barcelona: Editorial Masson S.A; 2002.

4. Watson J. Nursing Human science and Human Care: A Theory of Nursing New York: National League for Nursing. 1988: 27-35.

5. Benner P, Wrubel J. La Primacía del Cuidado: El estrés y el afrontamiento en la
Salud y Enfermedad. Editorial AdissonWesley; 1989.

6. Travelbee J. Intervención en Enfermería Psiquiátrica. 2a ed. OMS. OPS; 1982.

7. Daza de Caballero R, Torres AM, Prieto de Romano G. Análisis crítico del cuidado de enfermería: Interacción, participación y afecto. Index Enferm. 2005; 14(4849): 18-22.

8. Kash C. Habilidades y Competencias en la Interacción Enfermera-Paciente. New York: Nurse Red; 1986.

9. Henderson V. Naturaleza de la enfermería: reflexiones 25 años después. Madrid: Interamericana, McGraw-Hill; 1994.

10. Taylor S G. Teoría del Déficit de Autocuidado de Dorotea Orem. En: Marriner Tomey A, Raile Alligood M. Modelos y Teorías en Enfermería. 7a ed. Barcelona: Editorial Elsevier; 2008. Pp. 267-295.

11. Sakraida TJ. Modelo de la promoción de la salud de Nola Pender. En: Marriner Tomey A, Raile Alligood M. Modelos y Teorías en Enfermería. 7a ed. Barcelona: Editorial Elsevier; 2008. Pp. 452-471.

12. García M, Romero R. En: Daza de Caballero R, Torres A M, Prieto de Romano G. Análisis crítico del cuidado de enfermería: Interacción, participación y afecto. Index Enferm. 2005; 14(48-49): 18-22.

13. Código Sanitario. Decreto con Fuerza de Ley $\mathrm{N}^{\circ} 725$. [Internet] Actualizado a julio de 2000. Libro V, Artículo 113, inciso cuarto.. Hallado en: http://www. vertic.org/datasets/National\%20Legislation/Chile/Decree\%20Law\%20No.\%20 725\%201967.doc. [acceso 29 de agosto de 2009].

14. Ministerio de Salud. Norma General Administrativa $\mathrm{N}^{\circ} 19$. Gestión del Cuidado de Enfermería para la Atención Cerrada, Exenta $\mathrm{N}^{\circ} 1127$ [Internet] 2007. Hallado en: http://www.minsal.cl/juridico/RESOLUCION_1127_07.doc. [acceso el 30 agosto de 2009].

15. Grupo de Cuidado Facultad de Enfermería. Cuidado y Práctica de Enferme- 
ría. Bogotá: Editorial Unibiblios; 2000.

16. Zárate R. La Gestión del Cuidado de Enfermería. Index Enferm. 2004; 13(4445): 42-46.

17. Milos P, Larrain A, Simonetti M. Categorización de servicios de enfermería. Propuesta para asegurar una atención de calidad en tiempos de escasez de enfermeras. Cienc Enferm. 2009; 15(1): 17-24.

18. Ferro M. La gestión del cuidado en enfermería. Cuadernos de la Fundación Víctor Grífols i Lucas. 2005; 12(1): 1137.

19. Trevizan M, Mendes I, Fávero N, Melo M. Liderança e comunicação no cenário da gestão em enfermagem. Rev. LatinoAm Enfermagem. 1998; 6(5): 77-82.

20. Balsanelli A, Cunha I. Liderança no contexto da enfermagem. Rev. esc. enferm. USP. 2006; 40(1): 117-122.

21. Van-der Hofstadt CJ. Libro de las habilidades de comunicación. Cómo mejo- rar la comunicación personal: Ediciones Díaz de Santos. 2a ed. España; 2005.

22. Ayuso D, Grande R. La gestión de enfermería y los servicios generales en las organizaciones sanitarias. España: Ediciones Díaz de Santos; 2006.

23. Santos K, Silva M. Comunicação entre líderes e liderados: visão dos enfermeiros. Rev. esc. enferm. USP. 2003; 37(2): 97108.

24. Marriner Tomey A. Guía de Gestión y Dirección de Enfermería. 8 ${ }^{a}$ ed. Barcelona España: Elsevier; 2009.

25. Lamata F. Manual de Administración y Gestión Sanitaria. Madrid. España: Ediciones Díaz de Santos; 1998.

26. Gilles D. Gestión de Enfermería. Una aproximación a los sistemas. Barcelona: Masson-Salvat Enfermería; 1994.

27. Potter P, Perry A. Fundamentos de Enfermería. $5^{\mathrm{a}}$ ed. St. Louis: Elsevier Mosby; 2002 . 